Check for updates

Cite this: RSC Adv., 2018, 8, 904

Received 13th October 2017

Accepted 13th December 2017

DOI: 10.1039/c7ra11290d

rsc.li/rsc-advances

\section{Myelin-specific T cells carry and release magnetite PGLA-PEG COOH nanoparticles in the mouse central nervous system $\dagger$}

\begin{abstract}
M. M. D'Elios, ${ }^{\star a}$ A. Aldinucci, (D) ${ }^{\text {b } R . ~ A m o r i e l l o, ~}{ }^{b}$ M. Benagiano, ${ }^{a}$ E. Bonechi, ${ }^{b}$ P. Maggi, ${ }^{c}$ A. Flori, ${ }^{d}$ C. Ravagli, ${ }^{e}$ D. Saer, ${ }^{e}$ L. Cappiello, ${ }^{e}$ L. Conti, (D) ${ }^{f}$ B. Valtancoli, ${ }^{f}$ A. Bencini, (iD ${ }^{f}$ L. Menichetti, ${ }^{9}$ G. Baldi*e and C. Ballerini iD *b

Progress in nanotechnology has determined new strategies concerning drug delivery into the central nervous system for the treatment of degenerative and inflammatory diseases. To date, brain targeting through systemic drug administration, even in a nano-composition, is often unsuccessful. Therefore, we investigated the possibility of loading T lymphocytes with PGLA-PEG COOH magnetite nanoparticles (30 $\mathrm{nm})$, which can be built up to easily bind drugs and monoclonal antibodies, and to exploit the ability of activated $T$ cells to cross the blood-brain barrier and infiltrate the brain parenchyma. Iron oxide nanoparticles have been widely used in biomedical applications due to their theranostic properties and are therefore a well-established nanomaterial. The magnetite core is easily hybridized with polymeric compounds that may enhance the possibility of the nanoparticles entering cells with low phagocytic properties. Taking advantage of these material characteristics, after in vitro assessment of the viability and functionality of nano-loaded $\mathrm{MOG}_{35-55}$ specific $\mathrm{T}$ cells, we transferred cells containing the nanocargo into naïve mice affected by experimental autoimmune encephalomyelitis, an animal model of multiple sclerosis. By means of histological and immunohistological methods, we were able to identify the nano-loaded $T$ cells in the central nervous system. Our data demonstrated that $T$ cells containing nanomaterials hold the possibility of carrying and releasing nanoparticles in the brain.
\end{abstract}

\section{Introduction}

Drug delivery for the treatment of central nervous system (CNS) diseases has been substantially improved since the advent of nanotechnology. Indeed, the CNS is a difficult target for therapies delivered by the systemic route due to the comprehensive vascular organization of a physical and chemical barrier (the blood brain barrier, BBB) and to the absence of a classical lymphatic. ${ }^{1}$ This is the scenario faced in most neurodegenerative diseases, including progressive phases of multiple sclerosis (MS), a chronic immuno-mediated demyelinating disease of the CNS, in which the BBB is restored, or closed, while

${ }^{a}$ Department of Clinical and Experimental Medicine, University of Florence, Italy

${ }^{b}$ Department of Neuroscience, Psychology, Drug and Child Health, University of Florence, Italy. E-mail: clara.ballerini@unifi.it; Tel: +390552758252

'Department of Neurology, Hôpital CHU Brugmann-Université libre de BruxellesBruxelles-Be, Belgium

${ }^{d}$ Fondazione CNR Regione Toscana G. Monasterio, Pisa, Italy

${ }^{e}$ Research Center Colorobbia, Cericol, Colorobbia Consulting, Vinci, Florence, Italy. E-mail: baldig@colorobbia.it; Tel: +390571 709758

${ }^{f}$ Department of Chemistry Ugo Schiff, University of Florence, Italy

${ }^{g}$ Italian National Research Council (CNR), Pisa, Italy

$\uparrow$ Electronic supplementary information (ESI) available: ESI figures and methods. See DOI: $10.1039 / \mathrm{c} 7 \mathrm{ra} 11290 \mathrm{~d}$ compartmentalized inflammatory cell reactions are ongoing and triggering neurodegenerative processes. ${ }^{2}$ Localized clearing of the immune reaction and cure are dependent on the efficiency of CNS parenchyma penetration by the administered drug; therefore intrathecal delivery of immunosuppressants is a promising new approach for this more malign phase of the disease. ${ }^{3}$ For instance, Komori et al. recently investigated the effects of intrathecally-administered rituximab, a monoclonal antibody directed against CD20 that had no effect on clinical outcomes in MS progressive forms if delivered by the peripheral route. ${ }^{4} \mathrm{~A}$ decade of experience demonstrates that CNS direct drug administration is a possibility for anti-spastic therapies: for example, in patients resistant to orally administered Baclofen, intrathecal delivery was shown to be a good strategy in order to obtain an improvement in MS spasticity. ${ }^{5}$ We think that in MS, aside from the design of new drugs, the development of technologies promoting brain drug uptake in order to achieve therapeutic levels is becoming more and more crucial, as direct intrathecal delivery minimizes side effects and limits invasive procedures.

In recent years the development of nanotechnologies has provided a means for crossing the $\mathrm{BBB}$ and targeting the inflamed MS brain; many laboratories have developed nanocarriers for drug delivery and nano-vaccines constitute an 
efficient strategy to promote immune tolerance. ${ }^{6}$ Furthermore, nanomaterials have the ability to combine diagnostic with therapeutic approaches: ${ }^{7}$ ultra-small magnetite nanoparticles (USPIO) have been used as contrast medium in order to enhance MRI identification of CNS newly formed lesions in MS by visualizing macrophage cell infiltrates and different patterns of lesions ${ }^{8,9}$ Lesions in MS have been classified into 4 different patterns ${ }^{10}$ depending on the composition of cell infiltrate: demyelination, remyelination, oligodendrocyte loss and BBB damage. To date, there is a large ongoing effort in order to correlate histology data and neuroimaging data. We think that the future development of a MRI protocol able to spot NBRloaded $\mathrm{T}$ cells in MS active lesions may lead to the identification of those lesions characterized by the presence of $\mathrm{T}$ cells.

In the present work, we demonstrated that nanoparticles internalized by immune cells might be a promising strategy in order to deliver therapeutics into the brain. More specifically, we utilized nanoparticles feasible for future conjugation with drugs and exploited the ability of $\mathrm{T}$ lymphocytes to be loaded, to carry, and to cross the BBB and release these nanoparticles into the CNS. Our experimental design is based on antigen-specific $\left(\mathrm{MOG}_{35-55}\right)$ mice $\mathrm{T}$ cells efficiently loaded with nanobeads (PGLA-PEG-COOH plus a magnetite core; NBR) and then transferred i.v into $\mathrm{C} 57 \mathrm{Bl} / 6$ mice, naive or during the chronic phase of the animal model of MS -experimental autoimmune encephalomyelitis (EAE). Our research demonstrates that $\mathrm{T}$ cells loaded with NBR are able to migrate and effectively infiltrate the CNS tissue, where most importantly they were found in demyelinating lesions. Furthermore, we showed that in the CNS, the presence of utilized magnetite nanoparticles was identifiable by magnetic resonance imaging (MRI). Altogether, these findings provide exciting opportunities regarding the future of direct CNS MS lesions treatment.

\section{Experimental}

\section{Assembling nano-shells: NBR and NBR-Fluo}

Magnetite $\left(\mathrm{Fe}_{3} \mathrm{O}_{4}\right)$ nanoparticles $\left(\mathrm{NP}^{\mathrm{Fe}}\right)$ were synthesized as previously reported (Patent: Baldi et al., Magnetic Nanoparticles functionalized with cathecol, production and use thereof, $\mathrm{WO} /$ 2015104 664). ${ }^{11}$ Briefly, $40 \mathrm{~mL}$ of $\mathrm{NP}^{\mathrm{Fe}}$ suspended in diethylene glycol was sonicated for 1 hour in an ultrasound bath and then a solution of [N-(3,4-dihydroxyphenethyl)dodecanamide (DDA)] (1.09 g, $3.2 \mathrm{mmol})$ dissolved in $120 \mathrm{~mL}$ of ethanol was slowly added to the glycolic suspension. The system was again sonicated for 1 hour at room temperature. The solution was then diluted with $60 \mathrm{~mL}$ of ultrapure water, magnetically sedimented and dispersed in $90 \mathrm{~mL}$ of THF $\left(\mathrm{Fe}_{3} \mathrm{O}_{4}-\mathrm{DDA}\right)$. Then 440 milligrams of PGLA- $b$-PEG-COOH $(43-3 \mathrm{kDa}, 9.6 \mu \mathrm{mmol})$ was dissolved in $10 \mathrm{~mL}$ of THF and then added to $90 \mathrm{~mL}$ of $\mathrm{Fe}_{3} \mathrm{O}_{4}{ }^{-}$ DDA suspension (440 $\mathrm{mg}$ of $\mathrm{Fe}_{3} \mathrm{O}_{4}$ ). Fluorescent nanoparticles $\left(\mathrm{Fe}_{3} \mathrm{O}_{4}\right.$-DDA-Fluo) were achieved by adding a DMSO solution of Fluorescein 488-NHS (synthesized as reported within the ESI, Fig. S1 and methods $\dagger$ ), properly derivatized with 4-aminobutylphosphonic acid, to the $\mathrm{Fe}_{3} \mathrm{O}_{4}$-DDA suspension. The suspension was left reacting overnight at room temperature. The formation of NBR was achieved by the nanoprecipitation method: the organic phase was mixed with $900 \mathrm{~mL}$ of ultrapure water under vigorous stirring, maintaining a water : organic ratio of $10: 1$ with constant removal of the resulting solution. The mixture was then purified by washing with a phosphatebuffered solution and concentrated (Pellicon XL, cut-off 500 $\mathrm{kDa}$ ) to a final volume of $150 \mathrm{~mL}$ (concentration: $0.3 \%(\mathrm{w} / \mathrm{w})$ in $\mathrm{Fe}_{3} \mathrm{O}_{4}$ ); it was then filtered through a syringe filter (Millipore Sterivex, $0.22 \mu \mathrm{m}$, polyethersulfone membrane). Fluorescent NBR (NBR-Fluo) were prepared using the same method by substituting $\mathrm{Fe}_{3} \mathrm{O}_{4}$-DDA with $\mathrm{Fe}_{3} \mathrm{O}_{4}$-DDA-Fluo.

\section{Experimental animals}

Female C57BL/6 mice (Envigo RMS Srl, Italy) were bred in the CeSaL animal house of the University of Florence, in Makrolon cages, with free access to food and water and kept at $23^{\circ} \mathrm{C}$ with a 12 hours light/dark cycle. Every manipulation of the animals was executed according to the guidelines of the European community for animal care (DL 116/92, application of the European Communities Council Directive 86/609/EEC) and approved by the Committee for Animal Care (DGSAF, Italy, Authorization no. 514-2016-PR).

\section{EAE induction}

EAE was induced in 8 to 10 week-old female mice by s.c. injection of $200 \mathrm{mg}$ myelin oligodendrocyte glycoprotein $(M O G)_{35-55}$ peptide (MEVGWYRSPFSRVVHLYRNGK, purity 85\%; Espikem, Florence, Italy) in PBS, emulsified with an equal volume of complete Freund's adjuvant (CFA, Sigma) supplemented with $3 \mathrm{mg} \mathrm{mL}^{-1}$ Mycobacterium tuberculosis H37Ra (Difco Laboratories, USA). Bordetella pertussis toxin (300 ng) was administered by i.p. injection at days 0 and 2 post immunization (Calbiochem, Germany). EAE-induced mice were scored daily for clinical signs of disease.

\section{Generation of $\mathbf{M O G}_{35-55} \mathbf{T}$ cell lines (TCL)}

Lymphomonocytes were isolated from the spleen and by draining lymph nodes of immunized mice at 8 days postimmunization (dpi), by mechanical dissociation on nylon filters with $70 \mu \mathrm{m}$ pores (BD Falcon), followed by density gradient centrifugation (Biocoll, Biochrom AG). Cells were seeded in 96-well $U$ bottom plates (BD Falcon) at a density of 2 $\times 10^{5}$ per well with $10 \mu \mathrm{g} \mathrm{mL}^{-1}$ of $\mathrm{MOG}_{35-55}$ in complete medium (RPMI $1640+10 \%$ fetal bovine serum $+1 \%$ penicillinstreptomycin $+1 \%$ sodium pyruvate $+1 \%$ L-glutamine $+1 \%$ Hepes buffer, Sigma). A week later, cells were stimulated with 20 IU $\mathrm{mL}^{-1}$ of IL-2 (InvivoGen). After another 7 days, the experiment proceeded with the proliferation assay, according to the "split-well" method: $100 \mu \mathrm{L}$ of volume from the first plate was transferred to a second plate, divided into two volumes $(50 \mu \mathrm{L}$ per well). In one of the wells, we added $50 \mu \mathrm{L}$ of $\mathrm{MOG}_{35-55}$ and in the other one, $50 \mu \mathrm{L}$ of medium only. Then, $1 \times 10^{5}$ irradiated (6000 rad) spleen cells were placed in both. After $72 \mathrm{~h}$ of incubation at $37{ }^{\circ} \mathrm{C}, 5 \% \mathrm{CO}_{2}, 1 \mu \mathrm{Ci}$ of ${ }^{3} \mathrm{H}$-thymidine (Perkin Elmer) was added into each well. Then, the cells were transferred to a glass fibre filter for the proliferation analysis with the Cell Harvester (TomtecMacIII). The proliferation activity was 
measured as counts per minute (cpm) with a Trimux Microbeta 1450 counter (Wallac). We considered the test positive when $\Delta \mathrm{cpm}$ was $>1000 \mathrm{cpm}(\Delta \mathrm{cpm}=\mathrm{cpm}$ of stimulated wells $-\mathrm{cpm}$ of not-stimulated wells) and the stimulation index (SI) $>2$ (SI: cpm of stimulated wells/cpm of not-stimulated wells).

\section{Loading of $\mathrm{MOG}_{35-55}$ TCL with NBR nanoparticles}

MOG $_{35-55}$ TCL were incubated with NBR or NBR-Fluo, $(0.3 \%)$ at a density of $2 \times 10^{6}$ cells per mL in medium (RPMI $1640+10 \%$ fetal bovine serum $+1 \%$ penicillin-streptomycin $+1 \%$ sodium pyruvate $+1 \%$ L-glutamine $+1 \%$ Hepes buffer; all from Sigma) at $37^{\circ} \mathrm{C}$ for $2 \mathrm{~h}$; where indicated, NBR-Fluo loading was carried out in parallel at $4{ }^{\circ} \mathrm{C}$. Then, cells were washed 3 times and suspended in fresh medium for the next analysis. NBR-Fluo uptake by TCL was assessed by flow cytometry, evaluating the percentage of Fluorescein 488-positive cells and the related mean fluorescence intensity (MFI). MFI of samples loaded at $4{ }^{\circ} \mathrm{C}$ and $37{ }^{\circ} \mathrm{C}$ were compared by overlaying the corresponding FL-1 histograms; the number of events of all compared histograms were normalized based on the maximum peak value using the specific formatting histograms option of FCS Express6 software (DeNovo software, USA). For each sample, we acquired the same number of events. Dead cells, labelled with propidium iodide $\left(2.5 \mu \mathrm{g} \mathrm{mL}{ }^{-1}\right.$, Molecular Probes), were excluded from the analysis.

\section{Subcellular evaluation of NBR loaded TCL}

The $1 \times 10^{6}$ NBR-loaded TCL were centrifuged at $1500 \mathrm{rpm}$ for $5 \mathrm{~min}$ at RT, the supernatant was discarded and the cell pellet was fixed in Karnovsky solution; then it was embedded in epoxy resin. Samples were sectioned with an ultramicrotome in 50-60 nm slices. The sections were observed with TEM (Jeol, Japan). TEM analysis was performed on TCL immediately after NBR loading and upon 24 hours of stimulation in vitro with anti-CD3 $\varepsilon$ antibodies $1 \mu \mathrm{g} \mathrm{mL}{ }^{-1}$ (clone 17A2, eBioscience). To investigate the NBR-loaded $\mathrm{T}$ cell shape, a further evaluation was performed on $2 \times 10^{6}$ TCL loaded with NBRFluo. Cells were seeded on positively charged glass slides (Bioptica), fixed with PFA 4\% (Bioptica) for $15 \mathrm{~min}$ at RT, then washed with PBS and permeabilized with PBS/FBS 5\%/Triton $0.3 \%$ solution for $30 \mathrm{~min}$ at RT in the dark. F-actin distribution was evaluated in TCL loaded with NBR-Fluo, or not, by labelling with AlexaFluor 633 phalloidin (Thermo Fisher Scientific) for $20 \mathrm{~min}$ at RT in the dark, and then the slides were washed three times in PBS and closed with glass coverslips using mounting medium containing DAPI (Prolong Gold with DAPI, Life Technologies). Images were acquired using a Leica DMIRE2 confocal microscope (Leica Microsystems, Germany) equipped with a $\mathrm{He} / \mathrm{Ne} / \mathrm{Ar}$ laser source, using a Leica Plan Apo x 63/1.40 NA oil immersion objective. A series of optical sections $(1024 \times 1024$ pixels each; pixel size $200 \times$ $200 \mathrm{~nm}$ ) were taken at intervals of $0.3 \mu \mathrm{m}$. Confocal images were analysed using ImageJ software (National Institutes of Health [NIH], Bethesda, MD).

\section{Cell viability}

NBR-loaded TCL were analysed for cell viability immediately after loading and upon in vitro stimulation for 24 and 72 hours with $\mathrm{MOG}_{35-55} 10 \mu \mathrm{g} \mathrm{mL}{ }^{-1}$ in complete medium. Cell viability was assessed by flow cytometry using the Annexin V Apoptosis Detection Kit FITC (eBioscence, U.S.A.) and the CyFlow Space cytometer (Sysmex-Partec, Germany).

\section{Proliferation and cytokine production of $\mathrm{MOG}_{35-55}$ TCL loaded with NBR}

Proliferation activity of TCL loaded with NBR was tested by ${ }^{3} \mathrm{H}$ thymidine incorporation test as previously described, ${ }^{\mathbf{1 2}}$ after in vitro stimulation with $10 \mu \mathrm{g} \mathrm{mL}^{-1}$ of $\mathrm{MOG}_{35-55}$. Before adding ${ }^{3} \mathrm{H}$-thymidine, we collected $50 \mu \mathrm{L}$ of supernatant from each well for cytokine analysis. We measured IL-17, IFN $\gamma$, IL-4 and IL-10 production by Bioplex (Biorad), using the commercial kit Milliplex (MCYTOMAG-70K, Millipore) following the manufacturer's protocol.

\section{Expression of activation markers}

TCL, loaded or not with NBR, were stimulated with $\mathrm{MOG}_{35-55}$ $\left(10 \mu \mathrm{g} \mathrm{mL}^{-1}\right)$ and analyzed after 12 and 24 hours in order to evaluate the expression of CD25 (activation) and CD62L (a key regulator of migration, early-modulated during cell activation). Cells were stained (20 minutes RT in the dark) with the following fluorescent anti-mouse monoclonal antibodies (all from eBioscience): anti-CD25 PE (1: 100 diluted), anti-CD62L APC ( $1: 600$ diluted), anti-CD3 $\varepsilon$ FITC (1:100 diluted). Then the cells were analysed with a CyFlow Space cytometer (SysmexPartec, Germany).

\section{NBR release}

NBR-Fluo-loaded $\mathrm{MOG}_{35-55}$ TCL were incubated as previously described and then plated in 6-well plates coated, or not, with anti-CD3 $\varepsilon$ antibodies (clone 17A2, eBioscience; $1 \mu \mathrm{g} \mathrm{mL}{ }^{-1}$ ) at a density of 600.000 cells $/ 600 \mu \mathrm{L}$ in complete culture medium. Cells were collected at baseline immediately after NBR-Fluo incubation (T0) and at different time points after loading: 2, 4, 6, 18, 24, 48 and 72 hours later (T2-T4-T6-T18-T24-T48-T72) upon in vitro culture with or without $\mathrm{CD} 3$ stimulation. Unstained cells were used as a negative control. The NBR-Fluo release was evaluated by flow cytometry (CyFlow Space cytometer, Sysmex-Partec, Germany) following the decrease of Fluorescein 488 mean fluorescence intensity (MFI) over time. The analysis was performed using FCS Express 6 software (De Novo Software, USA). In parallel, we determined the quantity of $\mathrm{Fe}_{3} \mathrm{O}_{4}$ in the cellular pellets by ICP-OES (Varian $710 \mathrm{ES}$ ). At the same time, we evaluated the percentage of NBR-Fluo-positive cells and the related MFI by fluorescence microscopy. Briefly, cells were seeded $\left(10^{4}\right.$ cells per spot) on positively charged glass slides, fixed and permeabilized as previously described. Slides were closed with aqueous mounting medium with DAPI and observed using a fluorescence microscope (Olympus BX600, Japan). MFI was quantified by ImageJ software, measuring the optical density of the green fluorescence conjugated to NBR in 
15 randomly selected fields chosen from 3 different experiments.

\section{Passive transfer of NBR-loaded MOG $_{35-55}$ TCL in EAE and naïve mice}

NBR loaded TCL were diluted in saline buffer at a density of $1 \times$ $10^{7}$ cells per $\mathrm{mL}$ and $100 \mu \mathrm{L}$ of cell suspension was injected i.v. in C57BL/6 naïve mice ( 8 weeks old) and in EAE mice at $16 \mathrm{dpi}$, soon after the acute phase. We used 4 animals per group and we replicated the experiments three times. After 48 hours from passive transfer, mice were sacrificed and the brain and spinal cord were explanted in order to check the presence of NBRloaded $\mathrm{T}$ cells by histology/immunohistochemistry and post mortem MRI.

\section{Histology and immunohistochemistry}

Brain and spinal cord samples were fixed with 4\% PFA, paraffinembedded and sectioned with a microtome in $5 \mu \mathrm{m}$ slices. Slices were stained with Hematoxylin and Eosin (H\&E), Luxol Fast Blue (LFB) and Perls, following standard protocols, or selected for immunofluorescence labelling. Briefly, slices were treated with citrate buffer (citric acid $10 \mathrm{mM}, \mathrm{pH}=6$; Sigma) in a thermostatic bath at $99{ }^{\circ} \mathrm{C}$ for $20 \mathrm{~min}$ and washed in hot water at $99{ }^{\circ} \mathrm{C}$ for antigen retrieval, permeabilized with PBS/FBS 5\%/Triton $0.3 \%$ (Sigma) for $30 \mathrm{~min}$ at RT, labelled overnight at $4{ }^{\circ} \mathrm{C}$ with antimouse CD3 $\varepsilon$ antibodies (rabbit polyclonal, Abcam), and then diluted in permeabilization buffer $1: 100$. Then, slices were incubated for one hour in the dark at RT with the secondary antibodies AlexaFluor 488 anti-rabbit IgG (Life Technologies), and diluted $1: 400$ in permeabilization buffer. After 3 washes with PBS, the slices were closed with glass coverslips using mounting medium containing DAPI (Prolong Gold with DAPI, Life Technologies). Slides were observed by light or fluorescence microscopy (Leica Microsystems, Germany).

\section{Results and discussion}

\section{Production and characterization of magnetite nanobeads (NBR)}

TEM imaging of nanoparticles is reported in Fig. 1A. Furthermore, we reported SEM-FEG-STEM imaging of the inorganic core and surface (Fig. 1B and C). The mean hydrodynamic diameter and mean polydispersity index for NBR was determined by dynamic light scattering, and found to be 44.1 $( \pm 1.6) \mathrm{nm}$ and $0.17 \pm 0.02$, respectively, indicating a narrow size distribution. No major differences were detected for NBR-Fluo which showed an average hydrodynamic diameter of 41.0

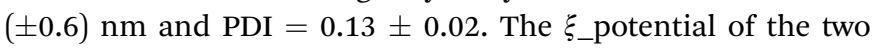
assemblies of particles was $-41 \mathrm{mV}$ and $-38 \mathrm{mV}$, respectively, for NBR and NBR-Fluo, confirming their stability. NBR-Fluo show an emission band centered at $520 \mathrm{~nm}$, typical of the fluorescent fluorophore (see ESI $\dagger$ ).

\section{Subcellular evaluation of MOG $_{35-55}$ TCL loaded with NBR}

Upload of NBR was qualitatively determined by TEM. Furthermore, we analysed the ultrastructure of NBR-loaded T cells,
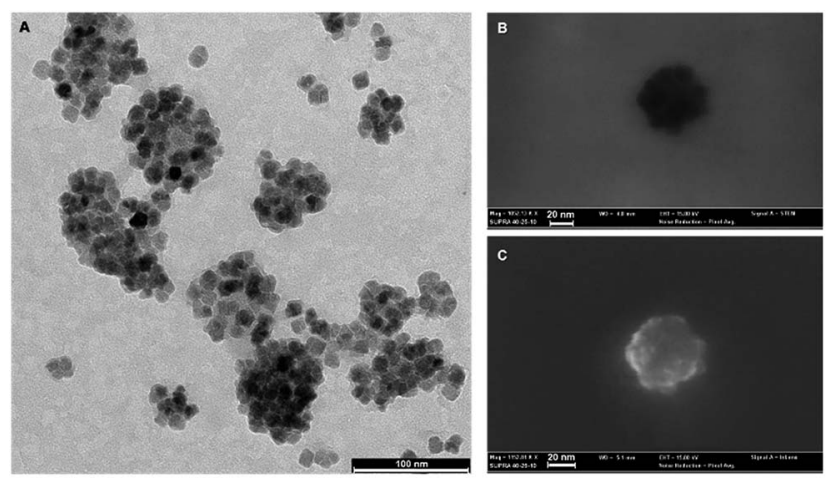

Fig. 1 Imaging of magnetite PGLA-PEG COOH nanoparticles. (A and B) TEM imaging of nanoparticles. (C) SEM-FEG-STEM imaging of the inorganic core and surface.

immediately after loading with nanoparticles (Fig. 2A(a and b)) and 24 hours later, upon in vitro stimulation with anti-CD3 antibody (Fig. 2A(c and d)). TEM micrographs show that the cells are healthy; the nanoparticles are distributed in the cytoplasm, mainly close to the cell membrane, and a few are spread within the nucleus; indeed, they are mostly concentrated in the cytoplasm, where they appear organized in the vesicle-like structures. During cell division, nanoparticles were in part transferred into the daughter cell (Fig. 2A(c and d)). To evaluate the possibility that NBR determines changes in $\mathrm{T}$ cell shape, we compared, using confocal microscopy, actin distribution (red) in control unloaded cells (Fig. 2B, first row panel) and cells loaded with NBR-Fluo (green) (Fig. 2B, second row panel). The figure shows that NBR does not affect cell shape, which remains circular, and there is a partial colocalization between the NBR-Fluo and actin (Fig. 2B, panel "merge"). Cell actin, compared to unloaded cells, showed a redistribution after NBR loading. In order to evaluate if loading of the NBR in T lymphocytes is a passive, or in part an active, event, we performed the NBR-Fluo loading protocol at $37^{\circ} \mathrm{C}$ and $4{ }^{\circ} \mathrm{C}$. As we have shown, at $4{ }^{\circ} \mathrm{C}$, when metabolic activity is reduced or there is no activity, loading of NBR is reduced in terms of quantity (MFI is threefold diminished), but loading still occurs and leads to $100 \%$ of cells being positive for NBR-Fluo (Fig. 2C).

In vitro analysis of NBR-loaded $\mathrm{MOG}_{35-55}$ TCL: viability, proliferation, cytokine production, and activation marker expression

First, we checked the viability of NBR-loaded TCL after 2 hours of incubation with nanoparticles and after in vitro stimulation with $\mathrm{MOG}_{35-55}$ for 24 and 72 hours (Fig. 3A). We did not find any significant variation in apoptotic and necrotic cell percentage in NBR-loaded TCL (black columns) compared to the controls (white columns).

The proliferative response to $\mathrm{MOG}_{35-55}$ stimulation was comparable in NBR-loaded TCL and controls (Fig. 3B, left). When we measured cytokine production, we detected a significantly decreased level of IFN $\gamma$ and IL17 $\left({ }^{*} p<0.05 ;{ }^{*} p<0.01\right)$ after $\mathrm{MOG}_{35-55}$ stimulation in NBR-loaded TCL compared to controls (Fig. 3B, right). Furthermore, we analysed the effect of 
A
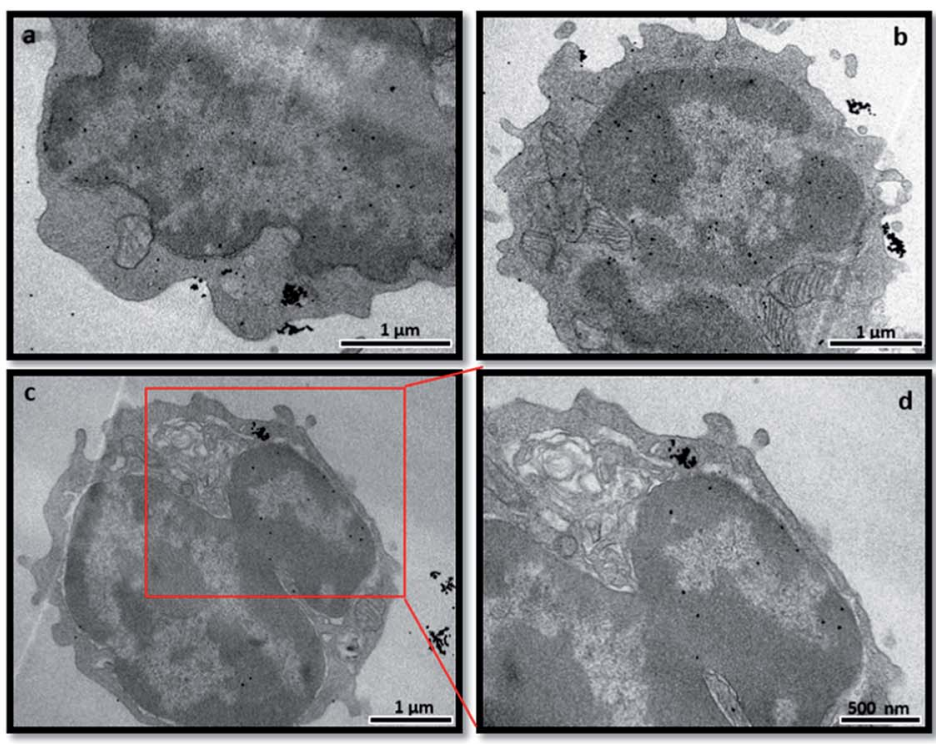

C

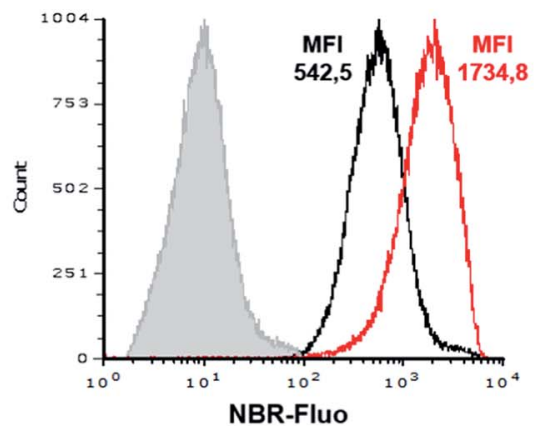

B
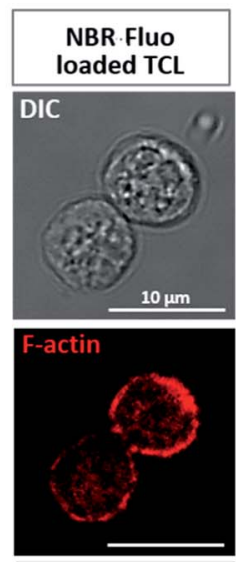

$-37^{\circ} \mathrm{C}$

$-4^{\circ} \mathrm{C}$

$\square$ unstained cells
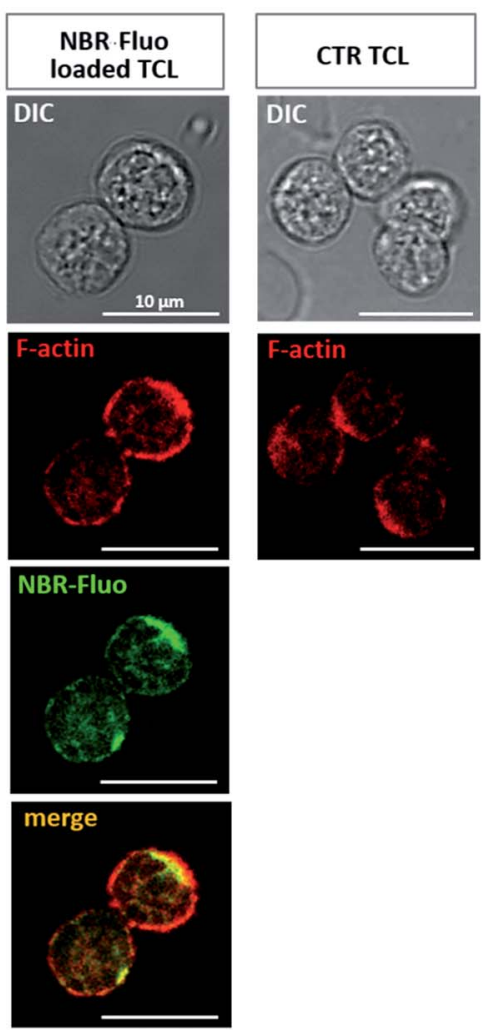

Fig. 2 Subcellular evaluation of NBR-loaded $M_{35-55} \mathrm{TCL}$. (A) TEM analysis of MOG $35-55$ TCL after incubation with iron-oxide NBR nanoparticles ( $a$ and $b$ ) or 24 hours later (c and d). Micrograph "(d)" represents a higher magnification of the image "(c)" (red square), showing a cell under division. Slice thickness: 50-60 nm. (B) MOG $35-55$ TCL loaded with NBR-Fluo analysed by confocal microscopy: NBR-Fluo (green) and Factin (red) (left panel). F-Actin distribution in control cells is reported in the right panel. One experiment is representative of three independent ones. Scale bar: $10 \mu \mathrm{m}$. (C) Flow cytometry evaluation of NBR-Fluo incorporation by $\mathrm{MOG}_{35-55} \mathrm{TCL}$ at $37^{\circ} \mathrm{C}$ (red histogram) or $4{ }^{\circ} \mathrm{C}$ (black histogram); grey histogram represents the intrinsic auto-fluorescence of unstained control cells. The numbers of events in the overlay histograms were normalized based on the maximum peak value. One experiment is representative of three independent ones.

NBR uptake on the cell surface expression of the activation markers CD25 and CD62L, both normally present after stimulation. CD25 is the IL2 receptor and CD62L is a lectin involved in cell migratory ability; they were evaluated upon $\mathrm{MOG}_{35-55}$ stimulation for 12 or 24 hours (Fig. 3C). The flow cytometry analysis indicated that activation of NBR T cells induces the expected up-regulation of CD25 and retains CD62L, and therefore $\mathrm{T}$ cells have the phenotype of normal active cells able to patrol the CNS parenchyma.

\section{In vitro analysis of MOG $_{35-55}$ TCL NBR uptake and release}

Before transfer of NBR-loaded TCL in vivo, we investigated for in vitro NBR release, by means of MFI determination in NBR-Fluoloaded cells and chemical determination of cell pellet $\mathrm{Fe}_{3} \mathrm{O}_{4}$ content. MFI value was measured (Fig. 4A) by flow cytometry immediately after NBR-Fluo cell incubation (T0) and after 2, 4, 6, 18, 24 and 72 hours; the green histograms in Fig. 4A show a reduction of MFI value from baseline to 72 hours of incubation compared to the control (unstained cells, white histogram). The evaluation of spontaneous or stimulationinduced release of nanoparticles by NBR-loaded TCL through measuring the $\mathrm{Fe}_{3} \mathrm{O}_{4}$ content gave the same result (Fig. 4B). After loading $2 \times 10^{6}$ antigen-specific T cells with NBR, we examined the cellular pellets, comparing them with the amount of $\mathrm{Fe}_{3} \mathrm{O}_{4}$ contained in an equal number of cells immediately after NBR loading. As shown in Fig. 3C, the $\mathrm{Fe}_{3} \mathrm{O}_{4}$ level decreased by half in the first 24 hours of culture, both in the stimulated cells and in the non-stimulated ones and continued to decrease slightly between 24 and 48 hours of culture, then remained stable between 48 and 72 hours. On the other hand, fluorescence microscopy (Fig. 4C) of the NBR-Fluo (green), and $T$ cells nuclei (DAPI, blue), shows that NBR-Fluo are maintained in almost $60 \%$ of the antigen-specific $\mathrm{T}$ cells from baseline to 72 hours of incubation. This determination was performed by observation of 5 areas per slide ( 3 slides for each time point), containing 25 cells per area for a total of three independent experiments. 

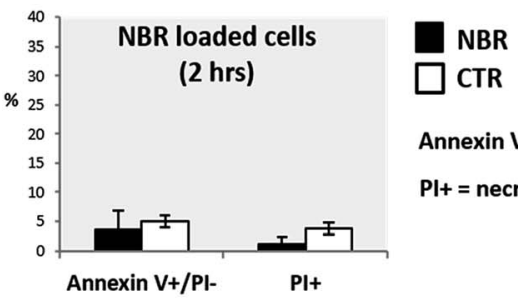

Annexin V+/PI- = apoptotic cells

$\mathrm{PI}+=$ necrotic cells
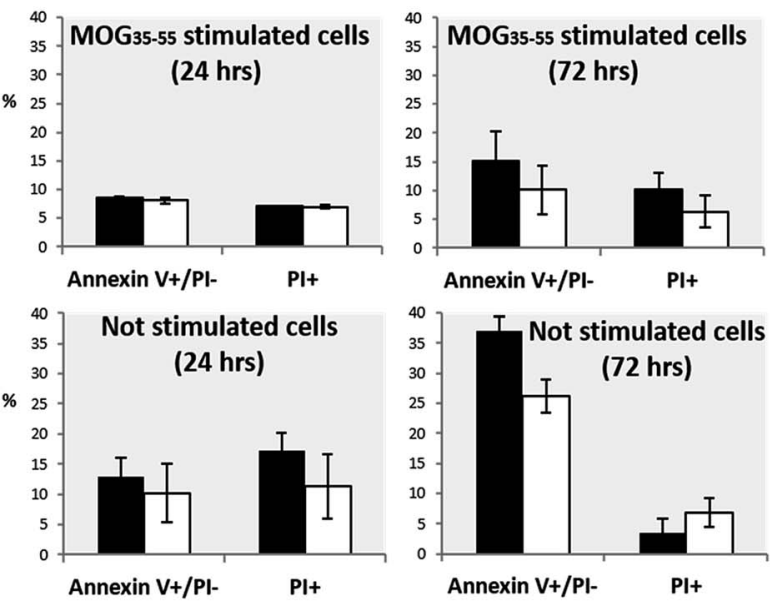
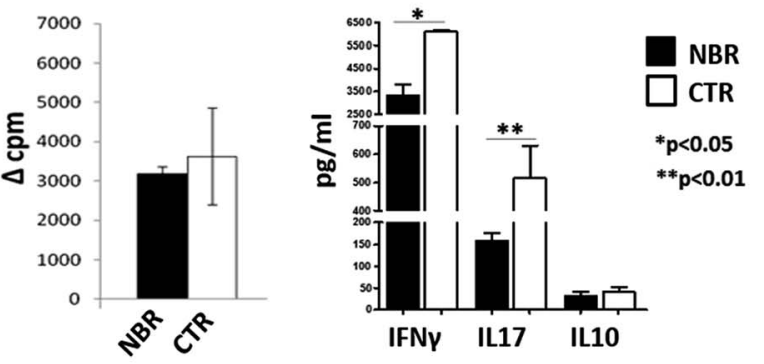

C
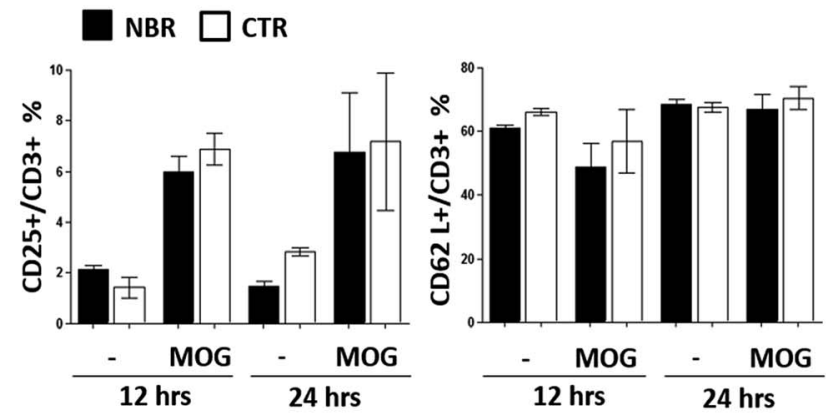

Fig. 3 Viability, proliferation, cytokine production and early activation markers of NBR-loaded TCL. (A) Analysis of the viability of NBR-loaded $\mathrm{MOG}_{35-55} \mathrm{TCL}$ compared to not-loaded cells (CTR) at different time points and experimental conditions: immediately after NBR loading (upper panel) and 24 and 72 hours later, upon in vitro stimulation with MOG $_{35-55}$ peptide (middle panel) or cultured with medium alone (lower panel). Mean value \pm SEM of three different experiments. PI = propidium iodide. (B) Left panel: antigen specific proliferative response of NBR loaded $M_{35-55} \mathrm{TCL}$ determined by ${ }^{3} \mathrm{H}$-thymidine incorporation assay and expressed as $\Delta \mathrm{cpm}$ (mean cpm observed in $\mathrm{MOG}_{35-55}$ stimulated cells mean cpm in not stimulated cells; $\mathrm{cpm}=$ counts per minute). Mean value \pm SEM of three experiments. Right panel: cytokine profile of NBRloaded $M_{35-55}$ TCL compared to not-loaded ones (CTR); IFN $\gamma$, IL17 and IL10 production was evaluated by Milliplex assays in supernatants of antigen-stimulated cells. Mean value \pm SEM of three experiments. (C) Flow cytometry analysis of early activation markers expression: CD25 (left) and CD62L (right) in MOG $35-55$ TCL loaded with NBR and not loaded (CTR) after 12 hours and 24 hours of in vitro culture with MOG $35-55$ peptide and in the absence of stimuli (-). Column graphs report the percentage of CD3 positive cells expressing CD25 and CD62L, respectively. Mean values \pm SEM of three independent experiments.

\section{Passive transfer of NBR loaded TCL into naïve mice}

After assessing viability, proliferative ability and phenotype of $\mathrm{MOG}_{35-55}$ TCL containing NBR in vitro, we studied in vivo ability of NBR-loaded TCL to migrate in CNS after i.v. cell transfer in naive animals, where BBB is intact. As reported in the methods section, mice were sacrificed 48 hours after the passive transfer. Fig. 5 illustrates Perls staining and reveals the presence of cells containing magnetite (blue cells) in the spinal cord (panel A) and brain sections (panels B and C). Focusing on brain tissue, we observed the presence of magnetite-positive cells spread in the hippocampus region (B) and within the cerebellum in the proximity of the fourth ventricle (C).

\section{Passive transfer of NBR-loaded TCL into EAE mice}

We then investigated whether NBR-loaded TCL were able to migrate into injured CNS tissue in EAE mice and if there was any colocalization with demyelinated lesions. The spinal cord designated to the histopathological analysis underwent a MRI investigation, and results (ESI, Fig. $2 \dagger$ ) were in agreement with Perls staining. In Fig. 6 we show the histological analysis in the thoraco-lumbar region of the spinal cord; within this area we observed infiltrates (H\&E) and demyelination (LFB), together with the presence of magnetite-positive cells (yellow arrows, Perls), identified in consecutive slices also as T cells by anti-CD3 immunofluorescence staining (white arrows).

Therapeutic delivery to the CNS during MS is challenging; direct lateral ventricular injections have been one option, although highly invasive and not feasible for treatments requiring frequent drug administration. ${ }^{4}$ Injections into cerebrospinal fluid are also a possibility, but diffusion in the brain parenchyma remains restricted. ${ }^{13}$ The direct use of nanomaterials able to physically interact with epithelial cells and shown to cross barriers, for example the BBB, could be a strategy in order to project new nano-drug formulations. ${ }^{14-16}$ Nonetheless, the absence of specificity of this interaction leads to a possible reduced efficacy in treatment of CNS diseases. Here, we decided to target CNS with autoreactive $\mathrm{T}$ cells, loaded with magnetite nanoparticles encapsulated in a PGLA-PEG-COOH copolymer (NBR) planned to easily cross the plasma membrane and to bind proteins, as antibodies. NBR dimensions make them suitable to cross membranes and internalize into cells following different pathways, ${ }^{17}$ becoming a cargo that may be transported and delivered in the brain. Activated $\mathrm{T}$ 
A

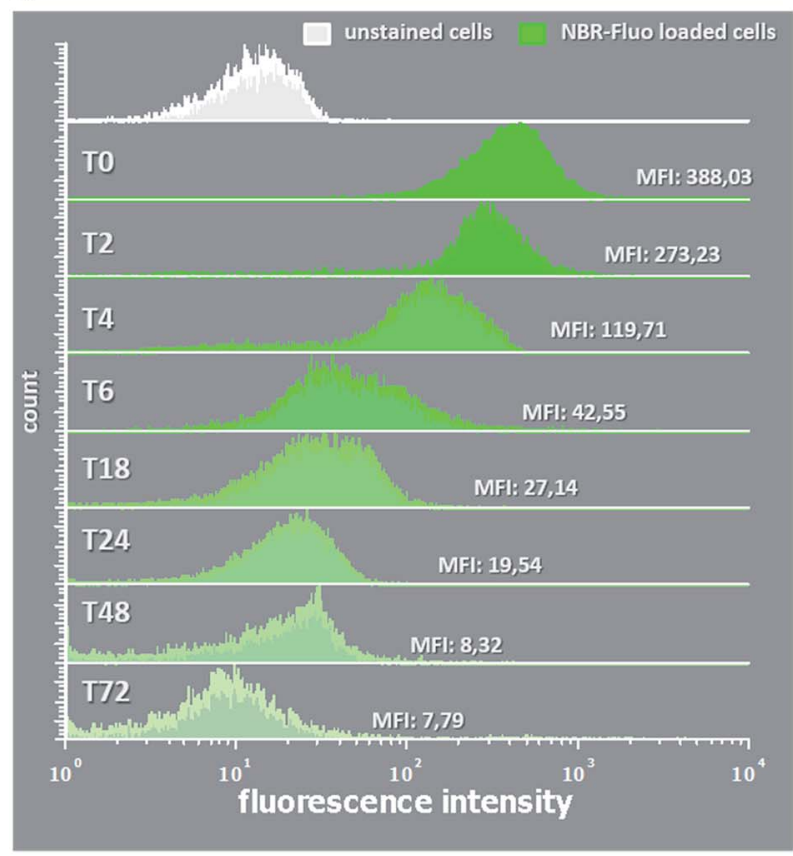

B

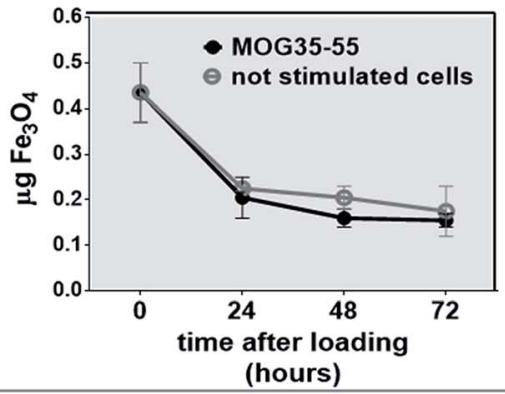

C

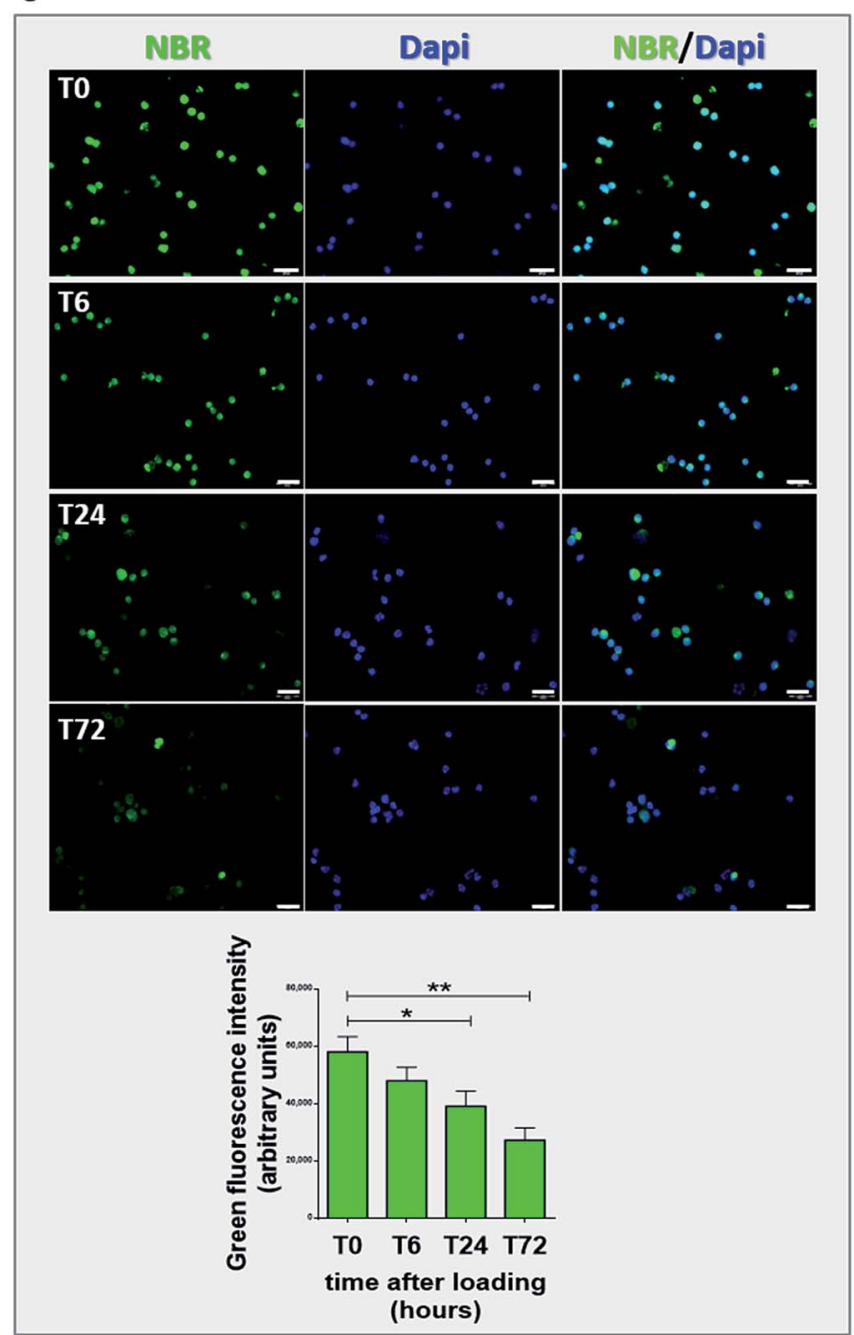

Fig. 4 Evaluation of nanoparticles release from NBR-loaded MOG $35-55$ TCL. (A) Green histograms show the mean fluorescence intensity (MFI) evaluated by flow cytometry of $\mathrm{MOG}_{35-55} \mathrm{~T}$ cells incubated with NBR-Fluo (Fluorescein 488) at different time points after loading: immediately after NBR loading (T0), and 2, 4, 6, 18, 24, 48 and 72 hours later (T2-T4-T6-T18-T24-T48-T72). Unstained cells were used as a negative control (white histogram). (B) Iron-oxide content expressed in $\mu \mathrm{g}$ of $\mathrm{Fe}_{3} \mathrm{O}_{4}$ measured by ICP-OES in MOG $35-55$ TCL 24, 48 and 72 hours after NBR loading, upon in vitro culture in the presence (black curve) or absence of CD3 stimulation (grey curve). Mean values \pm SEM of 3 experiments. (C) Upper panel: pictures of the same NBR-Fluo-loaded T cells analysed in panel A, taken using a fluorescence microscope at 4 different time points: T0-T6-T24-T72. Green: NBR-Fluo; blue: DAPI. Scale bar: $20 \mu \mathrm{m}$. One experiment is representative of 3 independent ones. Lower panel: quantification of the green fluorescence intensity in the NBR-Fluo-loaded T cells shown in the pictures. Column graph reports the average value \pm SEM of green fluorescence optical density measured by ImageJ in 15 randomly selected fields chosen from 3 different experiments ( $* p=$ $0.017 ; * * p=0.0045$, Mann-Whitney $t$ test).

lymphocytes cross the BBB and patrol brain parenchyma and, more important, depending on their antigen specificity, they will persist in the CNS with a peak at day 5 post-injection. ${ }^{18}$ The primary goal of this study was to investigate if NBR loaded CD4+ $\mathrm{T}$ lymphocytes, autoreactive for myelin antigens, were vital, functional and able to reach the CNS with an intact BBB and during the chronic phase of EAE.

We described how NBR are able to enter antigen-specific T lymphocytes without altering their shape and migratory properties, but partially modifying their cytokine profile towards a less inflammatory pattern. Electron microscopy imaging suggests that NBR locate in vesicle-like structures, and confocal microscopy shows that NBR are in close contact with actin, which resulted in a different distribution in loaded cells compared to controls. In previous work, we have demonstrated that actin cytoskeleton modifications regulate proinflammatory effector functions in myeloid dendritic cells; ${ }^{\mathbf{1 9 , 2 0}}$ here, we may speculate that a similar mechanism, driven by actin organization after NBR loading, is acting on $\mathrm{T}$ cells and is partially responsible of IL17 and IFN $\gamma$ down-regulation. In contrast with other reports concerning bare magnetite nanoparticles (5-30 $\mathrm{nm}),{ }^{21}$ NBR in part enters the nucleus, suggesting that this PGLA-covered nanomaterial is able to passively diffuse through all the membranes. Our data on cell loading at $4{ }^{\circ} \mathrm{C}$ indicates 

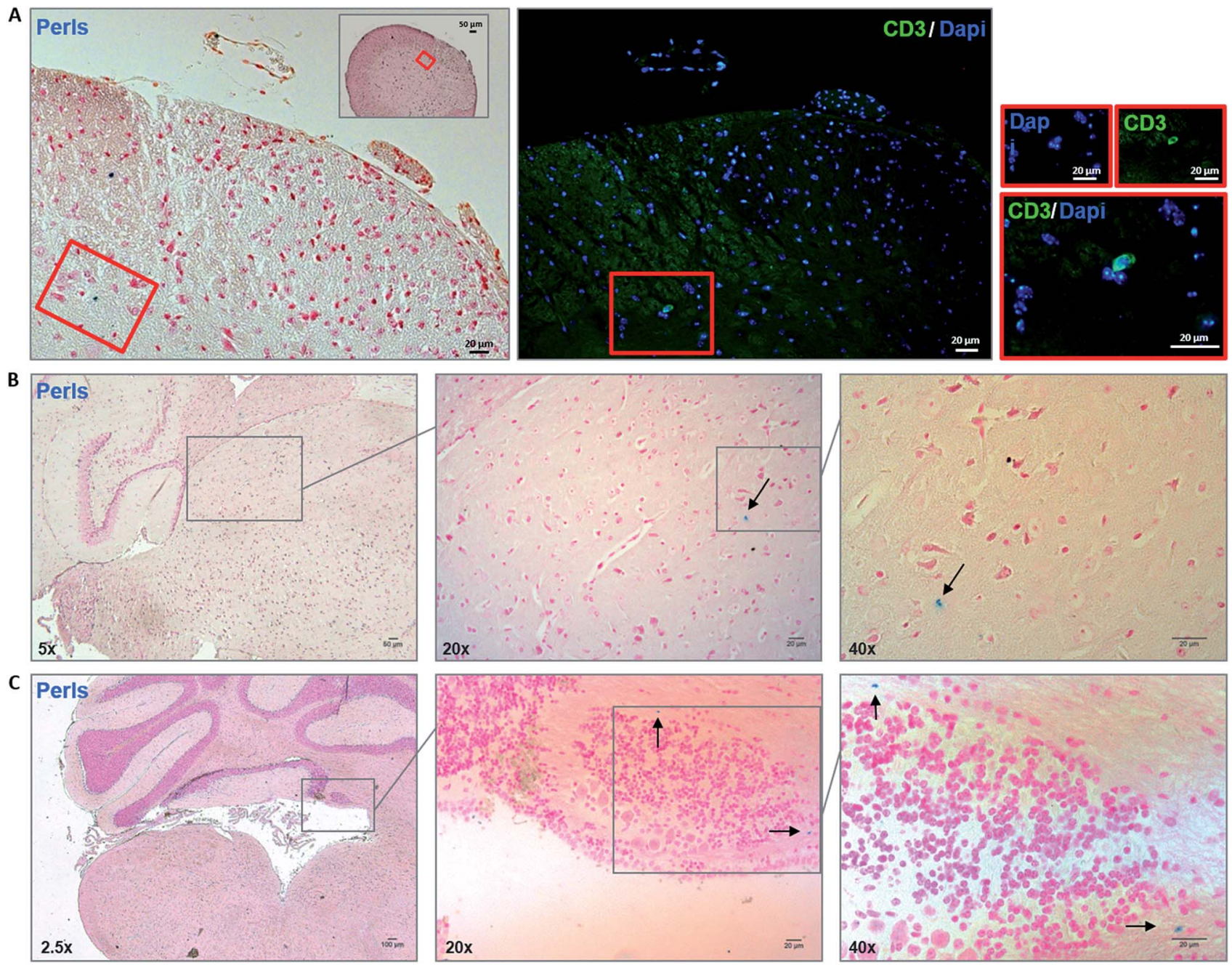

Fig. 5 Detection of magnetite-positive cells within the CNS of naive mice 48 hours after passive transfer of MOG $35-55$ TCL loaded with NBR. (A) Consecutive spinal cord sections ( $5 \mu \mathrm{m}$ thickness) stained with Perls (blue: iron-positive cells; red: nuclei; pink: cytoplasm) and immunolabeled with anti-CD3 fluorescent antibodies (green $=C D 3+T$ cells) and DAPI (blue = nuclei). Red rectangle maps a region where we found correspondence between Perls-positive cells and CD3 immunolabeling. ( $B$ and $C$ ) Perls staining revealed the presence of magnetite-positive cells (arrows) in the hippocampus region (B) and in the cerebellum, in the proximity of the fourth ventricle (C). Sections are observed at different magnifications as indicated $(2.5 x ; 5 x ; 20 x ; 40 x)$. Data are representative of three independent experiments.

that the mechanism of NBR cell entry is mostly passive, although an active component, an atypical macropinocytosis reported on HeLa cells loaded with SPION, ${ }^{22}$ might be present.

Our in vitro experiments demonstrated that nano-loaded cells start to gradually release the cargo during the first 24 hours. The apparent discrepancy between data from flow cytometry/chemical measures of NBR and fluorescence microscopy suggests that these cells are spontaneously releasing a part of the nano-cargo, but not all of it. Probably the flow cytometry and chemical analysis methods that we used for NBR content determination were not sufficiently sensitive, beneath a certain threshold of fluorescence, for a proper quantification of NBR-positive cells by flow cytometry. Notwithstanding the MFI data, fluorescence microscopy shows around $60 \%$ of cells still carrying NBR, even if to a lower extent, 72 hours after loading. Proliferation does not increase the spontaneous release of the nanomaterial, and we may hypothesize that during the proliferative response the magnetic particles are diluted in daughter cells, as suggested by an equal amount of NBR-positive cells in stimulated and not-stimulated protocols. These kinetics makes the system suitable for transport and delivery of therapies (e.g. anti CD20) designed to be effective in the periphery and in the CNS. In this perspective, we demonstrated that cells loaded with NBR cross the BBB in naive animals, where barrier permeability and integrity are normal, and reach the brain in 48 hours. Therefore, in naive mice, we confirmed that NBR-loaded cells were able to reach the brain parenchyma. Of note, the number of injected cells was far below the usual threshold that induces BBB damage..$^{23}$ On the other hand, thanks to their antigen specificity and activation state, they may stay and interact with neural cells. ${ }^{24}$ To further challenge this experimental delivery system, we injected NBRloaded $\mathrm{T}$ cells during the chronic phase of EAE, the animal model of multiple sclerosis. In these experiments, we suggest 

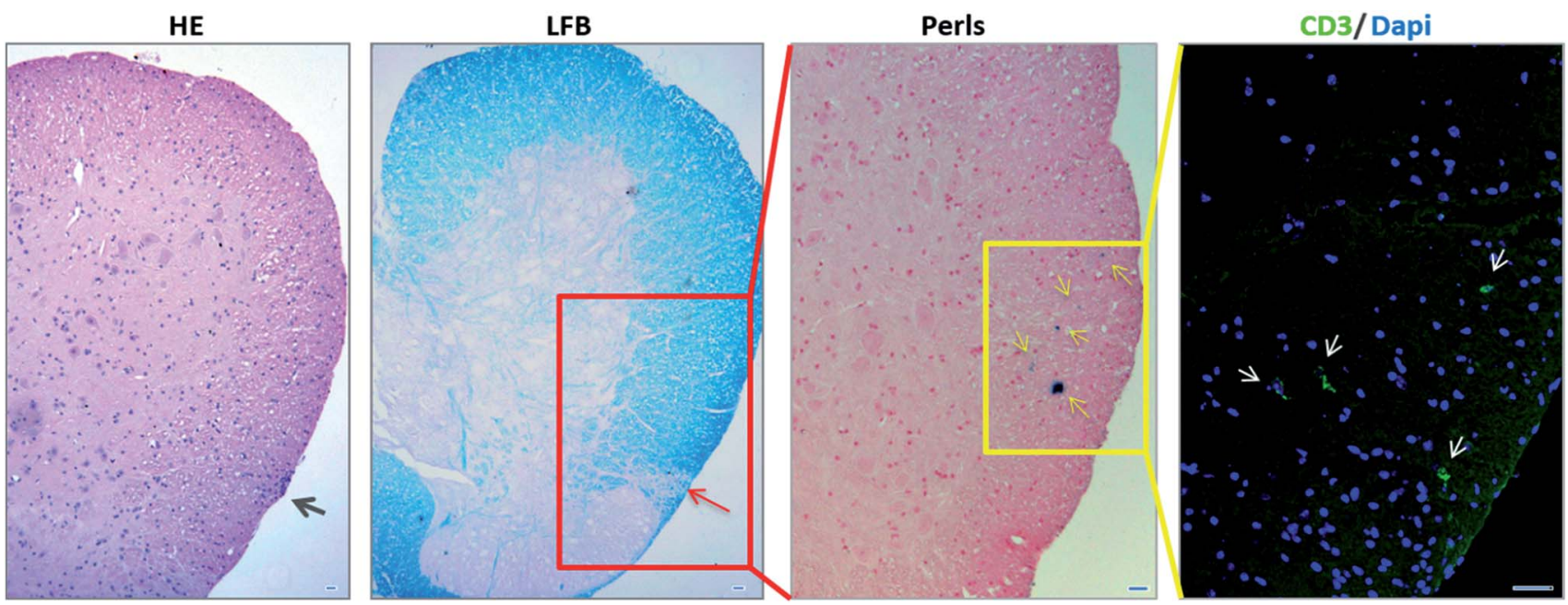

Fig. 6 Histological analysis of EAE spinal cord after the passive transfer of NBR-loaded TCL. Histological analysis at the thoracic-lumbar region of spinal cord. Consecutive spinal cord slices ( $5 \mu \mathrm{m}$ thickness) were stained with Hematoxylin/Eosin (HE) to reveal infiltrating immune cells (grey arrows), Luxol Fast Blue (LFB) to highlight demyelinating lesions (red arrows), Perls to detect the presence of magnetite-positive cells (yellow arrows) and anti-CD3 fluorescence immune-labelling to identify the presence of T cells (green, white arrows). Scale bars: $20 \mu \mathrm{m}$.

the possibility of tracing NBR cells, due to their ability to generate detectable signals in MRI, as previously described. ${ }^{25}$ Furthermore, the presence of NBR/cells in the CNS was confirmed by histology and immunohistochemistry; we found transferred cells containing NBR and NBR alone, probably released by the cells, also in demyelinated inflammatory areas 48 hours after injection. In fact, by this time antigen-specific $\mathrm{T}$ lymphocytes directed to a CNS antigen should be between the CNS perivascular space and the parenchyma, in an intact BBB scenario, or directly in the parenchyma when BBB permeability is altered. ${ }^{26}$

\section{Conclusions}

To the best of our knowledge, the use of $\mathrm{T}$ cells as carriers of nanoparticles has been previously assessed in cancer therapy, where nanoparticles were reversibly attached to activated CD8+ $\mathrm{T}$ cell surfaces ${ }^{27,28}$ and in autoimmunity, where poly(ethylene glycol)-functionalized hydrophilic carbon clusters specifically target $\mathrm{T}$ lymphocytes in the spleen in vivo and act as an antioxidant. ${ }^{29}$ Therefore, this is the first step towards the development of nano-loaded self-antigen-specific $\mathrm{T}$ lymphocytes as a therapy delivery system in the context of brain autoimmune disease.

\section{Conflicts of interest}

Cericol Research Center Colorobbia, Italy, provided nanoparticles, named NBR, used in this work.

\section{Acknowledgements}

We acknowledge Daniele Guasti for technical support in electron microscopy image. The present work was in part supported by Regione Toscana, project INSIDE, FESR 2014-2020.

\section{References}

1 B. Engelhardt, P. Vajkoczy and R. O. Weller, The movers and shapers in immune privilege of the CNS, Nat. Immunol., 2017, 18(2), 123-131.

2 R. Magliozzi, O. Howell, A. Vora, B. Serafini, R. Nicholas, M. Puopolo, et al., Meningeal B-cell follicles in secondary progressive multiple sclerosis associate with early onset of disease and severe cortical pathology, Brain, 2007, 130(4), 1089-1104.

3 A. Shirani, D. T. Okuda and O. Stüve, Therapeutic Advances and Future Prospects in Progressive Forms of Multiple Sclerosis, Neurotherapeutics, 2016, 13(1), 58-69.

4 M. Komori, Y. Chih Lin, I. Cortese, A. Blake, J. Ohayon and J. Cherup, Insufficient disease inhibition by intrathecal rituximab in progressive multiple sclerosis, Ann. Clin. Transl. Neurol., 2016, 3(3), 166-179.

5 M. Natale, S. D'Oria, V. V. Nero, E. Squillante, M. Gentile and M. Rotondo, Long-term effects of intrathecal baclofen in multiple sclerosis, Psychiatr., Neurol., Neurochir., 2016, 143, 121-125.

6 C. Ballerini, G. Baldi, A. Aldinucci and P. Maggi, Nanomaterial applications in multiple sclerosis inflamed brain, J. Neuroimmune Pharmacol., 2015, 10(1), 1-13.

7 M. S. Muthu, D. T. Leong, L. Mei and S. S. Feng, Theranostics - application and further development of nanomedicine strategies for advanced theranostics, Theranostics, 2014, $4(6), 660-677$.

8 V. Dousset, B. Brochet, M. S. Deloire, L. Lagoarde, B. Barroso, J. M. Caille and K. G. Petry, MR imaging of relapsing multiple sclerosis patients using ultra-small-particle iron oxide and compared with gadolinium, Am. J. Neuroradiol., 2006, 27(5), 1000-1005.

9 A. Crimi, O. Commowick, A. Maarouf, J. C. Ferré, E. Bannier, A. Tourbah, I. Berry, J. P. Ranjeva, G. Edan and C. Barillot, 
Predictive value of imaging markers at multiple sclerosis disease onset based on gadolinium- and USPIO-enhanced MRI and machine learning, PLoS One, 2014 Apr 1, 9(4), e93024.

10 C. F. Lucchinetti, W. Brück, M. Rodriguez and H. Lassmann, Distinct patterns of multiple sclerosis pathology indicates heterogeneity on pathogenesis, Brain Pathol., 1996, 6(3), 259-274.

11 D. Psimadas, G. Baldi, C. Ravagli, M. Comes Franchini, E. Locatelli, C. Innocenti, et al., Comparison of the magnetic, radiolabeling, hyperthermic and biodistribution properties of hybrid nanoparticles bearing $\mathrm{CoFe}_{2} \mathrm{O}_{4}$ and $\mathrm{Fe}_{3} \mathrm{O}_{4}$ metal cores, Nanotechnology, 2014, 25(2), 025101.

12 A. Aldinucci, L. Rizzetto, L. Pieri, D. Nosi, P. Romagnoli, T. Biagioli, et al., Inhibition of immune synapse by altered dendritic cell actin distribution: a new pathway of mesenchymal stem cell immune regulation, J. Immunol., 2010, 185(9), 5102-5110.

13 S. Mitragotri, P. A. Burke and R. Langer, Overcoming the challenges in administering biopharmaceuticals: formulation and delivery strategies, Nat. Rev. Drug Discov., 2014, 13(9), 655-672.

14 M. I. Setyawati, C. Y. Tay, B. H. Bay and D. T. Leong, Gold Nanoparticles Induced Endothelial Leakiness Depends on Particle Size and Endothelial Cell Origin, ACS Nano, 2017, 11(5), 5020-5030.

15 C. Y. Tay, M. I. Setyawati and D. T. Leong, Nanoparticle Density: A Critical Biophysical Regulator of Endothelial Permeability, ACS Nano, 2017, 11(3), 2764-2772.

16 M. I. Setyawati, C. Y. Tay, D. Docter, R. H. Stauber and D. T. Leong, Understanding and exploiting nanoparticles' intimacy with the blood vessel and blood, Chem. Soc. Rev., 2015, 44(22), 8174-8199.

17 L. Shang, K. Nienhaus and G. U. Nienhaus, Engineered nanoparticles interacting with cells: size matters, $J$. Nanobiotechnol., 2014, $12,5$.

18 R. Bernard-Valnet, L. Yshii, C. Quériault, X. H. Nguyen, S. Arthaud, M. Rodrigues, et al., CD8 T cell-mediated killing of orexigenic neurons induces a narcolepsy-like phenotype in mice, Proc. Natl. Acad. Sci. U. S. A., 2016, 113(39), 10956-10961.

19 A. Aldinucci, A. Turco, T. Biagioli, F. M. Toma, D. Bani, D. Guasti, et al., Carbon nanotube scaffolds instruct human dendritic cells: modulating immune responses by contacts at the nanoscale, Nano Lett., 2013, 13(12), 60986105.
20 A. Aldinucci, E. Bonechi, C. Manuelli, D. Nosi, E. Masini, M. B. Passani, et al., Histamine Regulates Actin Cytoskeleton in Human Toll-like Receptor 4-activated Monocyte-derived Dendritic Cells Tuning CD4+ T Lymphocyte Response, J. Biol. Chem., 2016, 291(28), 1480314814.

21 M. Yu, S. Huang, K. Jun Yu and A. Morss Clyne, Dextran and Polymer Polyethylene Glycol (PEG) Coating Reduce Both 5 and $30 \mathrm{~nm}$ Magnetite Nanoparticle Cytotoxicity in 2D and 3D Cell Culture, Int. J. Mol. Sci., 2012, 13(5), 5554-5570.

22 D. Hofmann, S. Tenzer, M. B. Bannwarth, C. Messerschmidt, S. F. Glaser and H. Schild, Mass spectrometry and imaging analysis of nanoparticle-containing vesicles provide a mechanistic insight into cellular trafficking, ACS Nano, 2014, 8(10), 10077-10088.

23 A. Smorodchenko, J. Wuerfel, E. E. Pohl, J. Vogt, E. Tysiak, R. Glumm, et al., CNS-irrelevant T-cells enter the brain, cause blood-brain barrier disruption but no glial pathology, Eur. J. Neurosci., 2007, 26(6), 1387-1398.

24 N. Kawakami, U. V. Nägerl, F. Odoardi, T. Bonhoeffer, H. Wekerle and A. Flügel, Live imaging of effector cell trafficking and autoantigen recognition within the unfolding autoimmune encephalomyelitis lesion, J. Exp. Med., 2005, 201(11), 1805-1814.

25 S. A. Anderson, J. Shukaliak-Quandt, E. K. Jordan, A. S. Arbab, R. Martin, H. McFarland, et al., Magnetic resonance imaging of labeled T-cells in a mouse model of multiple sclerosis, Ann. Neurol., 2004, 55(5), 654-659.

26 A. S. Archambault, J. Sim, M. A. Gimenez and J. H. Russell, Defining antigen-dependent stages of $\mathrm{T}$ cell migration from the blood to the central nervous system parenchyma, Eur. J. Immunol., 2005, 35(4), 1076-1085.

27 S. A. Rosenberg, J. C. Yang, R. M. Sherry, U. S. Kammula, M. S. Hughes, G. Q. Phan, et al., Durable complete responses in heavily pretreated patients with metastatic melanoma using T-cell transfer immunotherapy, Clin. Cancer Res., 2011, 17(13), 4550-4557.

28 L. Wayteck, K. Breckpot, J. Demeester, S. C. De Smedt and K. Raemdonck, A personalized view on cancer immunotherapy, Cancer Lett., 2014, 352(1), 113-125.

29 R. Huq, E. L. Samuel, W. K. Sikkema, L. G. Nilewski, T. Lee, M. R. Tanner, et al., Preferential uptake of antioxidant carbon nanoparticles by $\mathrm{T}$ lymphocytes for immunomodulation, Sci. Rep., 2016, 6, 3380. 